\title{
Transmittance Simulation of Surface Textured Fluorine Doped Tin Oxide (FTO) for Dye Sensitized Solar Cell (DSSC) Application
}

\author{
Kusumandari*, Risa Suryana \\ Dept. of Physics, Faculty of Mathematic and Natural Sciences, Sebelas Maret University \\ Surakarta 57126, Indonesia \\ E-mail address: kndari@gmail.com
}

\begin{abstract}
The aim of this research is to simulate the transmittance of fluorine doped tin oxide (FTO) for dye sensitized solar cell (DSSC) application. Simulation was performed using finite difference time domain (FDTD) method based on Maxwell equation for flat and nano cone periodically surface textured of FTO. The simulation was done for 500 and $600 \mathrm{~nm}$ of incident plane wave wavelength. As a result, the transmittance power for nano cone surface textured is higher than flat FTO.
\end{abstract}

Keywords: DSSC, surface textured FTO, Simulation, FDTD

\section{Introduction}

Energy crisis has become a serious problem for many countries in the world due to limitation and unrenewable of energy source. Therefore, renewable energy sources such as solar energy are considered as a feasible alternative. So far, intensive research activities have resulted in attention-grabbing to the different classes of organic and inorganic based solar cells. ${ }^{1}$ Dye Sensitized Solar Cell (DSSC) is one of type solar cell which based on photo electrochemical process. The DSSC sandwich structure consist of transparent conducting (TCO) glass substrate and $\mathrm{TiO}_{2}$ as working electrode, dye as absorbent of light, electrolytes as a medium of exchange electron and $\mathrm{Pt}$ or carbon as counter electrode. ${ }^{2}$

Currently, the efficiency of DSSC is around $12.3 \%$ which is still far lower than silicon solar cell. ${ }^{3}$ Many attempts have been made to improve the efficiency of DSSC by modify of $\mathrm{TiO}_{2}$ structure such as using porous or nanotube of $\mathrm{TiO}_{2}{ }^{4}$ Another way is nano engineering of TCO to improve the light harvesting through enhance the light transmittance and scattering. Besides that, the surface texturing of TCO may also provide a direct scheme to enhance the interfacial surface area and hence power conversion efficiency. Application of surface texturing on the surface of silicone amorphous apparently managed to increase the efficiency of solar cells. ${ }^{5-7}$ In the DSSC, Wang et al. ${ }^{8}$ observe that the hemisphere of indium doped tin oxide (FTO) increase scattering of light. However, the effect of surface texturing of TCO in DSSC has not been fully investigated. Therefore, the purpose of this study is to develop a model which would be able to predict the optical characteristics of a thin surface textured FTO layer on glass. FTO is a kind of TCO which low cost, indium free, stable at high temperatures and have acidic and hydrogen eenvironments. ${ }^{8}$ This simulation was done with finite difference time domain (FDTD) method using OptiFDTD software. ${ }^{9}$ Using this simulation, the effect of nano cone surface patterns on the total transmission of light through the sample is then considered.

\section{Method}

In this study, FDTD method was used to numerically solve Maxwell's equations in three dimension. The optical simulations were used to analyze electromagnetic wave propagation in flat and nano cone surface textured of FTO substrate.

Maxwell's equation for homogenous medium: ${ }^{10}$ 


$$
\begin{aligned}
& \nabla x \vec{E}=-\mu \frac{d \vec{H}}{d t} \rightarrow\left\{\begin{array}{l}
\frac{\partial E_{z}}{\partial y}-\frac{\partial E_{y}}{\partial z}=-\mu \frac{\partial H_{x}}{\partial t} \\
\frac{\partial E_{x}}{\partial z}-\frac{\partial E_{z}}{\partial x}=-\mu \frac{\partial H_{y}}{\partial t} \\
\frac{\partial E_{y}}{\partial x}-\frac{\partial E_{x}}{\partial y}=-\mu \frac{\partial H_{z}}{\partial t}
\end{array}\right. \\
& \nabla x \vec{H}=\epsilon \frac{d \vec{E}}{d t} \rightarrow\left\{\begin{array}{l}
\frac{\partial H_{z}}{\partial y}-\frac{\partial H_{y}}{\partial z}=\epsilon \frac{\partial E_{x}}{\partial t} \\
\frac{\partial H_{x}}{\partial z}-\frac{\partial H_{z}}{\partial x}=\epsilon \frac{\partial E_{y}}{\partial t} \\
\frac{\partial H_{y}}{\partial x}-\frac{\partial H_{x}}{\partial y}=\epsilon \frac{\partial E_{z}}{\partial t}
\end{array}\right.
\end{aligned}
$$

In 3D simulations, the simulation domain is a cubic box with the space steps are Dx, Dy, and Dz, and in $\mathrm{x}$, $\mathrm{y}$, and $\mathrm{z}$ directions respectively.

Each field components of electromagnetic wave is presented by $\operatorname{Ex}(\mathrm{i}, \mathrm{j}, \mathrm{k}), \operatorname{Ey}(\mathrm{i}, \mathrm{j}, \mathrm{k}), \operatorname{Ez}(\mathrm{i}, \mathrm{j}, \mathrm{k}), \mathrm{Hx}(\mathrm{i}, \mathrm{j}, \mathrm{k})$, $\mathrm{Hy}(\mathrm{i}, \mathrm{j}, \mathrm{k})$, and $\mathrm{Hz}(\mathrm{i}, \mathrm{j}, \mathrm{k})$. The field components position in Yee's Cell are shown in Fig. 1. ${ }^{9}$

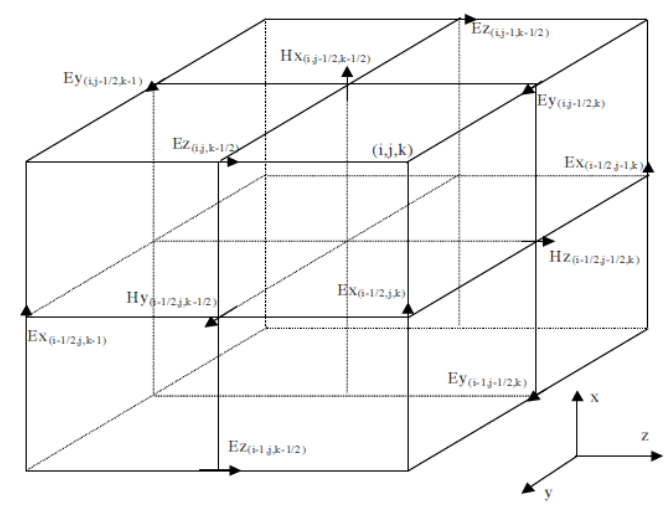

Fig. 1. Propagation of the electric and magnetic field vector component about cubic unit cell of Yee space lattice. ${ }^{9}$

Figure 2 show the flowchart of simulation. The simulation was done in three steps. First, we build the layout and define the parameters of object such as refraction index, thickness, width and length of sample. In this study we use FTO with refraction index of 1.92 and $300 \mathrm{~nm}$ of thickness. The width of simulation area of FTO is $500 \mathrm{~nm}$. The time step of simulation was set up for 1000. Simulations were done for flat and nano cone surface textured of FTO. The last process is analyzing the post data using OptiFDTD analyzer

Then we applied plane wave on the sample. Second, we start the simulation for $\mathrm{n}$ iteration. The $\mathrm{H}$ and $\mathrm{E}$ field were update interleaved during running simulation.

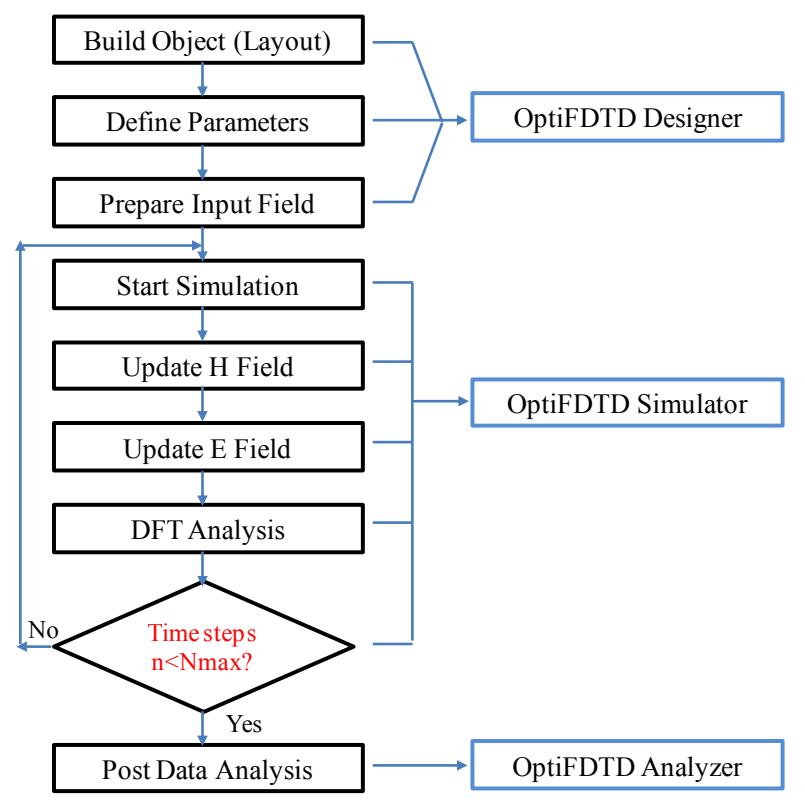

Fig. 2. The flowchart of simulation using OptiFDTD software. ${ }^{9}$

\section{Result and Discussion}

Numerical simulations using OptiFDTD based on FDTD method was performed in order to calculate the transmittance in the flat and nano cone surface textured of FTO. Figure 3 show the 3D layout of flat and nano cone surface textured of FTO. As illustrated in Figure 3 , the plane wave was come perpendicular from glass to FTO toward negative $\mathrm{z}$ axis. In this study, we use assumption that glass transmits all of incident plane wave. The wavelengths of plane wave are 500 and 600 nm.

Distribution of transmittance power intensity in y axis direction for (a) flat and (b) nano cone surface textured of FTO was shown in Fig. 4. Figure 4(a) shows the propagation of wave light in the flat surface FTO. There is no scattering was introduced. In other hand, Nano cone surface scatter the light in random direction as shown in Fig. 4(b). Interference between light scattered resulting strong intensity. Figure 5 shows corresponding total transmittance power before, along and after passing the FTO layer. It can be seen that the light intensity passed the nano cone surface textured is higher than flat surface. In the DSSC application, the propagation of scattered wave in the $\pm X$ directions increases the path length inside the active area ( $\mathrm{TiO} 2$ and Dye). Therefore, it helps the increase in conversion efficiency of DSSC. 


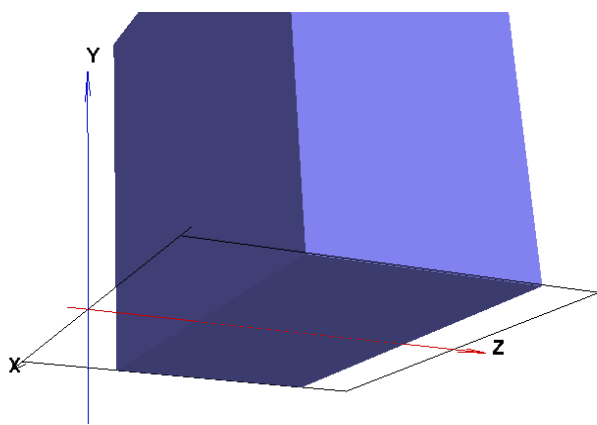

(a)

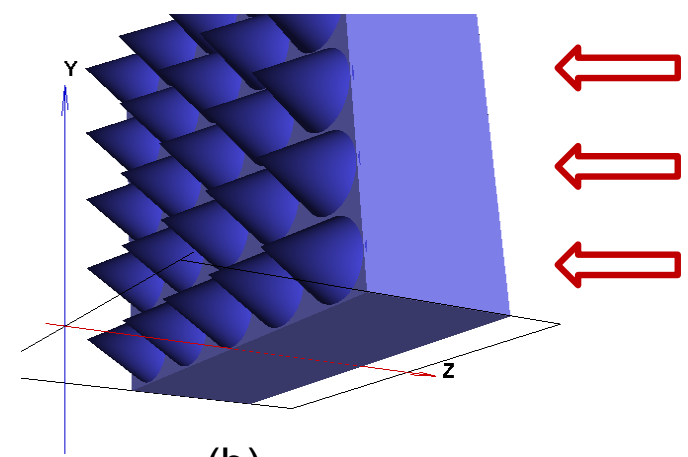

(b)

Fig. 3. The 3D layout of (a) flat and (b) nano cone surface textured of FTO. Red arrows indicate incident plane wave from glass to FTO.

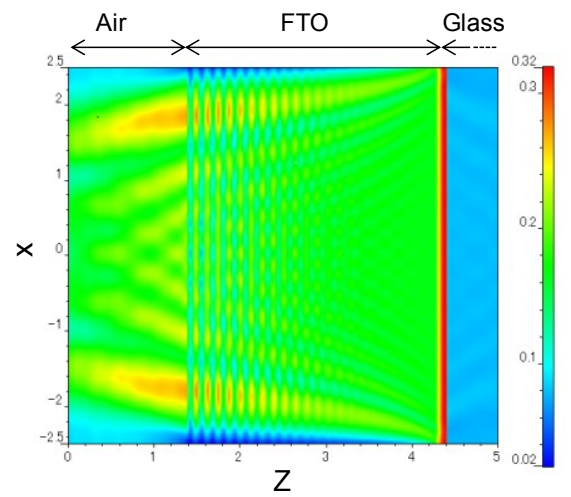

(a)

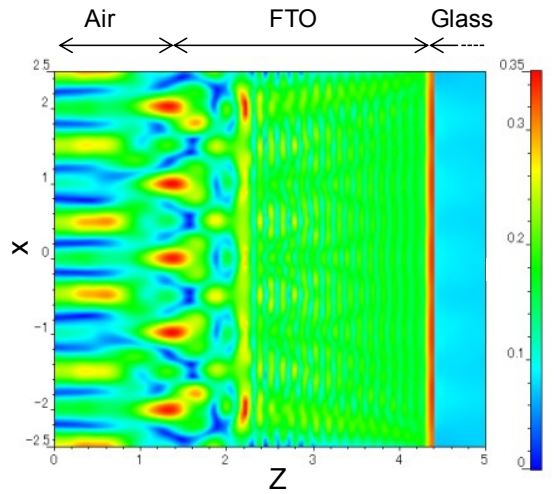

(b)

Fig. 4. The transmittance power distribution of $500 \mathrm{~nm}$ plane wave passing across glass/FTO/air interface for (a) flat and (b) nano cone surface textured of FTO.

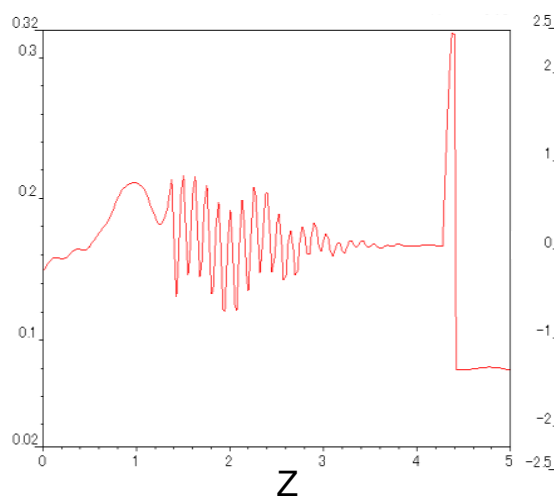

(a)

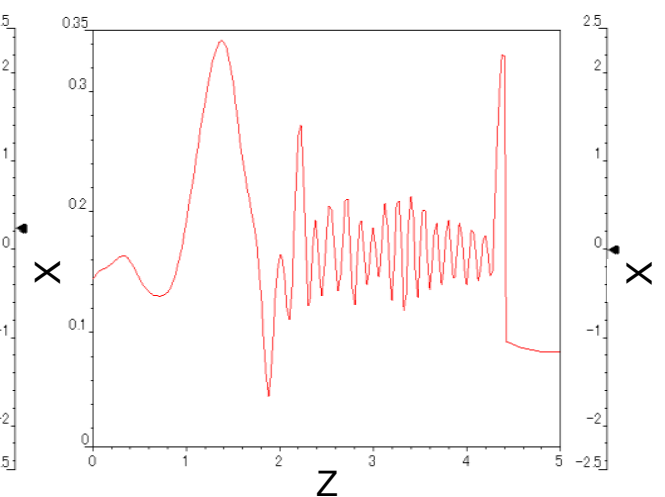

(b)

Fig. 5. Corresponding total transmittance power of $500 \mathrm{~nm}$ plane wave passing across glass/FTO/air interface for (a) flat and (b) nano cone surface textured of FTO.

As comparison, we also perform simulation for 600 $\mathrm{nm}$ of incident plane wave. Figure 4 show (a) the transmittance power distribution and (b) corresponding total transmittance. This result agree with previous that nano cone surface textured of FTO will enhance the transmittance of light. 


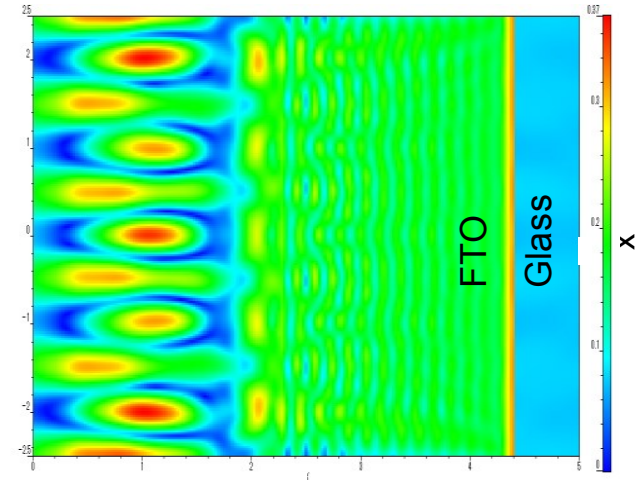

Z

(a)

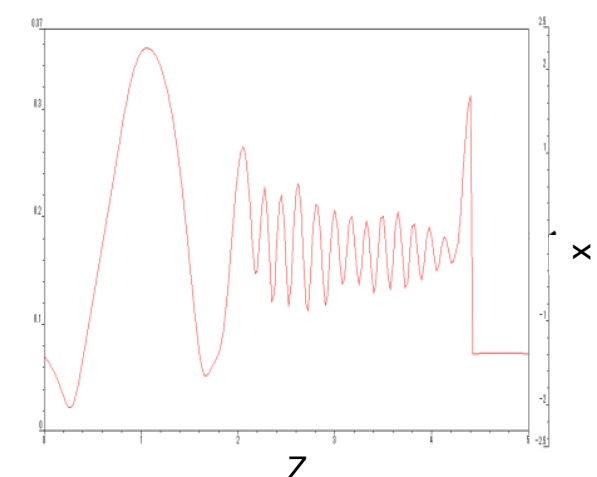

(b)

Fig. 4. (a) The transmittance power distribution and (b) Corresponding total transmittance power of $600 \mathrm{~nm}$ plane wave passing across glass/FTO/air interface for nano cone surface textured of FTO.

\section{Conclusion}

The simulation of nano cone surface textured of FTO on light transmittance for DSSC application was investigated using FDTD method. The nano cone surface textured exhibit significant differences compare to the flat surface resulting in an increased light scattering. The propagation of scattered light wave enhances power conversion in active layer which is expected to be viable scheme for achieving improved performance of DSSC.

\section{References}

1. K. Ebrahim Jasim, Dept. of Physics, University of Bahrain Kingdom of Bahrain, www.intechopen.com.

2. M. Gratzel, Dye Sensitized Solar Cell, J. Photochem. and Photobio. C: Photochem. Review (4) (2003), pp. 145 $-153$.

3. B. E. Hardin, H. J. Snaith and M. D. McGehee, The Renaissance of Dye-Sensitized Solar Cells, Nature Photonics (6) (2012), pp. 162-169.

4. X. Chen and S.S. Mao, Titanium Dioxide Nanomaterials : Synthesis, Properties, Modifications, and Applications, Chem. Rev. 107 (7) (2007), pp. 2891 2959.

5. V. Javanov, U. Palanchoke, P. Magnus, H. tiebig, J. Hiipkes, P. Sichanugrist, M. Konagai, S. Wiesendanger, C. Rockstuhl and D. Knipp, Light Trapping in Periodically Textured Amourphous Silicon Thin Film Solar Cells Using Realistic Interface Morphologies, Optics Express S4 (21) (2013), pp. 595-606.
6. C.H. Hsu, C. Bttaglia, C. Pahud, Z. Ruan, F.j. Haug, S. Fan, C. Ballif and Y. Cui, High Efficiency Amorphous Silicon Solar Cell on a Periodic Nanocone Back Reflector, Adv. Energy Mater. (2) (2012), pp. 628-633.

7. D. Domine, F.-J. Haug, C. Battaglia and C. Ballif, Modelling of Light Scattering from Micro- and Nanotextured Surfaces, J. Appl. Phys. 107 (044504) (2010), pp. 1-8

8. F. Wang, N.K. Subbaiyan, Q. Wang, C. Rochford, G. Xu, R. Lu, A. Elliot, F. D'Souza, R. Hui and J. Wu, Development of Nanopatterned Fluorine-Doped Tin Oxde Electrodes for Dye-Sensitized Solar Cells with Improved Light Trapping, ACS Appl. Mater. Interfaces (4) (2012), pp. 1565-1572.

9. OptiFDTD Technical Background and Tutorials, FDTD Photonic Simulation Software. http://optiwave.com/category/optifdtd-manuals/optifdtdbackground-and-tutorials/

10. H.J. Pain, The Physics of Vibration and Waves, 3rd edn. (John Wiley \& Sons Limited, New York, 1987). 